\title{
Three easily-implementable changes reduce median door-to-needle time for intravenous thrombolysis by 23 minutes
}

Demi $\operatorname{Tran}^{1+}{ }^{\text {, Zhu Zhu }}{ }^{1,2+}$, Mohammad Shafie ${ }^{1}$, Hermelinda Abcede ${ }^{1}$, Dana Stradling ${ }^{1}$ and Wengui $\mathrm{Yu}^{1,3^{*}}$ (D)

\begin{abstract}
Background: The benefit of intravenous thrombolysis (IVT) for acute ischemic stroke is time dependent. Despite great effort, the median door-to-needle time (DNT) was $60 \mathrm{~min}$ at the United States stroke centers. We investigated the effect of a simple quality improvement initiative on DNT for IVT.

Methods: This is a single-center study of patients treated with IVT between 2013 and 2017. A simple quality improvement initiative was implemented in January 2015 to allow the Stroke team to manage hypertension in the emergency room, to make decision for IVT before getting blood test results unless patients were taking oral anticoagulants, and to give IVT in the CT suite. Baseline characteristics, DNT and outcomes at hospital discharge were compared between pre- and post-intervention groups.

Results: Ninety and 136 patients were treated with IVT in pre- and post-intervention groups, respectively. The rate of IVT was significantly higher in the post-intervention group ( $20 \%$ vs. $14.4 \%, p=0.007$ ). The median DNT with interquartile range (IQR) was reduced significantly by $23 \mathrm{~min}(63[53-81]$ vs. 40[29-53], $p<0.001)$ with more patients in the post-intervention group receiving IVT within $60 \mathrm{~min}(81.6 \% \mathrm{vs} .46 .7 \%)$ and $45 \mathrm{~min}(64.0 \% \mathrm{vs} .17 .8 \%)$. There was no significant difference in symptomatic intracerebral hemorrhage rate (1.5\% vs. 1.1\%), modified Rankin Scale 0-1 (29.4\% vs. 23.3\%), and hospital mortality (7.4\% vs. 6.7\%) between the 2 groups.
\end{abstract}

Conclusions: Three easily-implementable quality improvement initiative increases IVT rate and reduces DNT significantly without increasing the rate of IVT-related complications in our comprehensive stroke center.

Keywords: Acute ischemic stroke, Intravenous thrombolysis, Door-to-needle time, Quality improvement

\section{Introduction}

Intravenous thrombolysis (IVT) with tissue-type plasminogen activator (tPA) is the proven medical therapy for acute ischemic stroke (AIS), with faster administration resulting in better outcomes $[1,2]$. However, the diagnosis and treatment of AIS are often delayed for various reasons, including lack of pre-notification, unclear last-known-well (LKW) time, waiting for blood test results, lag in getting CT scan and reports of imaging findings, holdup in mixing tPA or transportation between emergency room (ER) and CT scan suite, management of uncontrolled hypertension, performing CT

\footnotetext{
* Correspondence: wyu@uci.edu

${ }^{\dagger}$ Demi Tran and Zhu Zhu contributed equally to this work.

'Department of Neurology, University of California, Irvine, CA, USA

${ }^{3}$ Orange, USA

Full list of author information is available at the end of the article
}

angiography and CT perfusion, and determination of eligibility [3-10].

Researchers at Helsinki University Central Hospital in Finland were able to implement measures to reduce delays and cut the median door-to-needle time (DNT) to $20 \mathrm{~min}$ with interquartile range (IQR) $14-32 \mathrm{~min}$ [4]. The Helsinki protocol was successfully replicated at the Royal Melbourne Hospital to reduce DNT to 25 (IQR, 19-48) minutes during business hours (8 AM to $5 \mathrm{PM}$ Monday-Friday) in 2012 [5]. A few hospitals in Canada and Netherlands were also able to reduce median DNT to $25-37 \min [6-9]$.

In contrast, the quality improvement endeavors have not worked out very well in the United States (U.S.). Despite the launch of American Heart Association (AHA)/American Stroke Association (ASA) Target: Stroke initiatives in 2010 
and comprehensive stroke center (CSC) certification by the Joint Commission in 2012 [10, 11], the IVT rate and median DNT at the U.S. stroke centers remains suboptimal. A study of AIS patients registered in Get With The GuidelinesStroke from October 2012 to April 2015 showed that the median DNT was 60 min with only 50\% patients treated within $60 \mathrm{~min}$ [12]. The Target: Stroke phase II was launched in April 2014. The median DNT from 888 surveyed hospitals between June 2014 and April 2015 was still 56 (IQR, 42-75) minutes [13]. In a recent study comparing stroke care and outcomes between CSCs and primary stroke centers (PSCs) in the U.S. from 2013 to 2015, the median DNT was 52 (IQR, 39-70) minutes at CSCs and 60 (IQR, 47-83) minutes at PSCs [14]. The IVT rates were only 14.3 and $10.3 \%$, respectively.

It appears that centralized hospital system in developed countries were able to implement quality improvement initiatives efficiently [4-9]. The healthcare system in the U.S. is decentralized with higher average annual IVT volume in CSCs than in PSCs (40 vs. 22) [14]. Due to overall low annual volumes and labor-intensive code stroke protocols, the stroke centers in the U.S., including CSCs and PSCs, appear to have significant logistic restraints in implementing comprehensive quality improvement initiatives 24/7 [11-13].

Due to limited numbers of vascular neurologists and insufficient staff support, our stroke center was unable to implement some of the Stroke: Target phase 1 strategies during 2010 and 2014. We started to focus on the 3 easily-implementable changes in January 2015 to improve stroke care at our CSC. This study aimed to explore the effect and safety of this simple initiative.

\section{Methods}

\section{Patients and design}

This is a single-center study approved by the University of California Irvine Institutional Review Board. Acute Ischemic stroke is characterized by the sudden loss of blood circulation to an area of the brain, resulting in a corresponding loss of neurologic function. Consecutive patients with LKW within $4.5 \mathrm{~h}$ were evaluated for thrombolysis. Patients receiving IVT at the University of California Irvine CSC between January 2013 and December 2017 were included. A simple quality improvement protocol was developed in January 2015: 1) To allow the stroke team to manage hypertension in the ER promptly to keep BP goal $<185 / 110 \mathrm{mmHg}$. Patient was initially treated with labetalol or hydralazine $10 \mathrm{mg}$ IV pro re nata and then IV nicardipine infusion at $2.5-15 \mathrm{mg} /$ hour to keep steady BP control. Vital signs were measured every $15 \mathrm{~min}$. 2) To make decision for IVT before getting blood test results (complete blood count, comprehensive metabolic panel, cardiac enzymes and coagulation) unless patients were taking anticoagulants. All patients were carefully interviewed and examined to rule out history of coagulopathies, thrombocytopenia, and severe metabolic abnormalities before intravenous thrombolysis and t-PA would be immediately stopped when any potential contraindication for IVT was found during thrombolysis. All patient got finger stick for glucose measurement in the ambulance or Emergency Department (ED). Severe hypoglycemia was excluded before thrombolysis through finger stick glucose measurement. The benefit and risk of IVT were discussed with patients and/or their relatives, and written informed consent was not required American Heart Association guidelines [1]. 3) To give IVT in the CT suite.

The patients were divided into pre-intervention (January 2013 to December 2014) and post-intervention (January 2016 to December 2017) groups, with one-year washout period (January 2015 to December 2015) allowing for the full implementation of the initiative.

The following information was collected and compared between the pre- and post- intervention groups: age, gender, past medical history (hypertension, diabetes, hyperlipidemia), National Institutes of Health Stroke Scale (NIHSS) score at admission, DNT, symptomatic intracranial hemorrhage $(\mathrm{sICH})$, in-hospital mortality, and modified Rankin Scale (mRS) at hospital discharge. $\mathrm{sICH}$ was defined as parenchymal hemorrhage type 2 (dense blood clot exceeding 30\% of the infarct volume with substantial space-occupying effect) on $\mathrm{CT}$ within $36 \mathrm{~h}$ [2].

\section{Statistical analysis}

Continuous variables were described by mean \pm standard deviation or median with interquartile range (IQR) based on the results of normality testing. Categorical variables were expressed by counts with percentages. Baseline characteristics and outcomes at discharge were compared between pre- and post- intervention groups by $\mathrm{t}$ test or Wilcoxon rank-sum test for continuous variables and $x^{2}$ test for categorical variables. The proportions of $\mathrm{sICH}$, functional independence (mRS $0-1$ ), poor outcome (mRS 5-6), and in-hospital mortality were further compared between the 2 groups using multivariate logistic regression analysis after adjusting for age, hypertension, diabetes, hyperlipidemia and baseline NIHSS score. Analyses were performed using SPSS software (IBM, Version 23). A 2-tailed value of $P<0.05$ was considered statistically significant.

\section{Results}

A total of 1305 patients with AIS were admitted to our medical center during the study period and 294 of them received and completed IVT. After excluding 68 patients treated during the transitional year of the quality improvement initiative, there were 90 patients in pre-intervention 
group (from January 2013 to December 2014) and 136 in post-intervention group (from January 2016 to December 2017). The demographics and treatment benchmarks of the 2 groups are shown in Table 1. Compared with preintervention group, significantly more patients were treated with IVT in the post-intervention group $(20.0 \%$ vs. $14.4 \%$; $\mathrm{OR}=1.39 ; p=0.007$ ). There was no difference in patient age, gender, history of hypertension or diabetes between the 2 groups. The post-interventional group had significantly higher rate of hyperlipidemia and lower NIHSS scores at admission than pre-intervention group. The patients with minor stroke (NIHSS $\leq 4$ ) appeared to be more likely to receive IVT in the post-intervention group $(27.2 \%$ vs. $10.0 \%, p<0.001)$.

The median DNT was reduced by 23 min from 63 (IQR, $53-81$ ) minutes in the pre-intervention group to 40 (IQR, $29-53)$ minutes in the post-intervention group $(p<0.001)$, with a trend of continuous improvement from 2013 to 2017 (Fig. 1). In addition, significantly more patients in the post-intervention group received IVT within $60 \mathrm{~min}$ $(81.6 \%$ vs. $46.7 \%, p<0.001)$ and $45 \mathrm{~min}(64.0 \%$ vs. $17.8 \%$, $p<0.001)$ than in the pre-intervention group. Of note, there was no significant difference in DNT between patients with minor (NIHSS $\leq 4$ ) and major (NIHSS >4) stroke (median $50 \mathrm{~min}$, IQR $25-75 \mathrm{~min}$ vs. median 50 min, IQR 36-71 $\mathrm{min} ; p=0.317$ ).

The rates of sICH and functional outcomes at hospital discharge are summarized in Table 2. There was no significant difference in the rates of $\mathrm{sICH}(1.5 \%$ vs. $1.1 \%)$, functional independence (mRS $0-1,29.4 \%$ vs. $23.3 \%$ ), and hospital mortality $(7.4 \%$ vs. $6.7 \%)$ between the 2 groups. There was a trend of better functional outcome (more patients with mRS $0-1$ and significantly less patients with mRS 5-6) in the post-intervention group (Table 2 and Fig. 2). However, in the multi-variate regression models, there was insignificant difference between the 2 groups after adjusting for age, hypertension, hyperlipidemia, diabetes and NIHSS score at admission.

\section{Discussion}

With a simple and easily-implementable quality improvement initiative, we have increase IVT rate from 14.4 to $20.0 \%$ and reduced median DNT by $23 \mathrm{~min}$ to $40 \mathrm{~min}$. Our results are better than recently reported benchmarks of $14.3 \%$ IVT rate and 52 min DNT at the 134 CSCs in the U.S. [14].

Despite significant improvement, our results are still suboptimal compared with the benchmarks from centralized Hospital Systems in other developed countries [4-9]. For example, the hospital district of Helsinki and Uusimaa has a population of 1.6 million and a centralized regional emergency medical service (EMS) [4, 15]. All patients deemed as candidates for stroke therapies are transported with high priority and pre-notification to the Helsinki University Hospital, which is the only 24/7 neurology service to provide care for AIS. As a high volume and centralized Stroke center, the Helsinki University Hospital was very efficient due to thorough training for all EMS and ED staff, and long-standing experience $[4,15]$.

In contrast, our CSC, the only academic medical center in Orange County, California, is one of the 9 stroke receiving centers serving a population of 3.19 million [16]. In such decentralized healthcare system, it is very challenging for all of the 9 stroke receiving centers to implement comprehensive protocols to achieve the fastest DNT for IVT $[4-11,14]$.

Our quality improvement initiative is easilyimplementable, effective and safe. Uncontrolled hypertension is one of the most frequently reported factors causing delayed DNT $[5,8,17]$. In a single center study, uncontrolled hypertension was associated with more than 30 min delay in DNT [16]. Per AHA/ASA

Table 1 Demographics and clinical features of patients in the pre-intervention and post-intervention groups

\begin{tabular}{|c|c|c|c|c|}
\hline Variables & Pre-intervention & Post-intervention & OR $(95 \% \mathrm{Cl})$ & $p$ value \\
\hline Age & $70 \pm 16$ & $71 \pm 17$ & - & 0.745 \\
\hline Male & $44(48.9)$ & $68(50.0)$ & $1.04(0.61-1.78)$ & 0.870 \\
\hline Hypertension & $62(68.9)$ & $107(78.7)$ & $1.67(0.91-3.06)$ & 0.097 \\
\hline Diabetes & $22(24.4)$ & 46 (33.8) & $1.58(0.87-2.87)$ & 0.132 \\
\hline Hyperlipidemia & $29(32.2)$ & $62(45.6)$ & $1.76(1.01-3.07)$ & 0.045 \\
\hline $\mathrm{SBP} \geq 185 / 110 \mathrm{mmHg}$ & $21(23.3)$ & $41(30.1)$ & $1.44(0.81-2.54)$ & 0.215 \\
\hline NIHSS & $14(8-21)$ & $7(4-16)$ & - & $<0.001$ \\
\hline $\mathrm{NIHSS} \leq 4$ & $9(10.0)$ & $37(27.2)$ & $3.36(1.53-7.38)$ & 0.002 \\
\hline DNT & $63(53-81)$ & $40(29-53)$ & - & $<0.001$ \\
\hline $\mathrm{DNT}<60 \mathrm{~min}$ & $42(46.7 \%)$ & $111(81.6 \%)$ & $5.08(2.79-9.26)$ & $<0.001$ \\
\hline DNT $<45$ min & $16(17.8 \%)$ & 87 (64.0\%) & $8.20(4.31-15.3)$ & $<0.001$ \\
\hline
\end{tabular}

Data are $\mathrm{n}(\%)$, mean \pm standard deviation, or median (interquartile range)

OR Odds ratio, $\mathrm{CI}$ Confidence interval, SBP Systolic blood pressure, NIHSS National Institues of Health Stroke Scale, DNT Door-to-needle time 


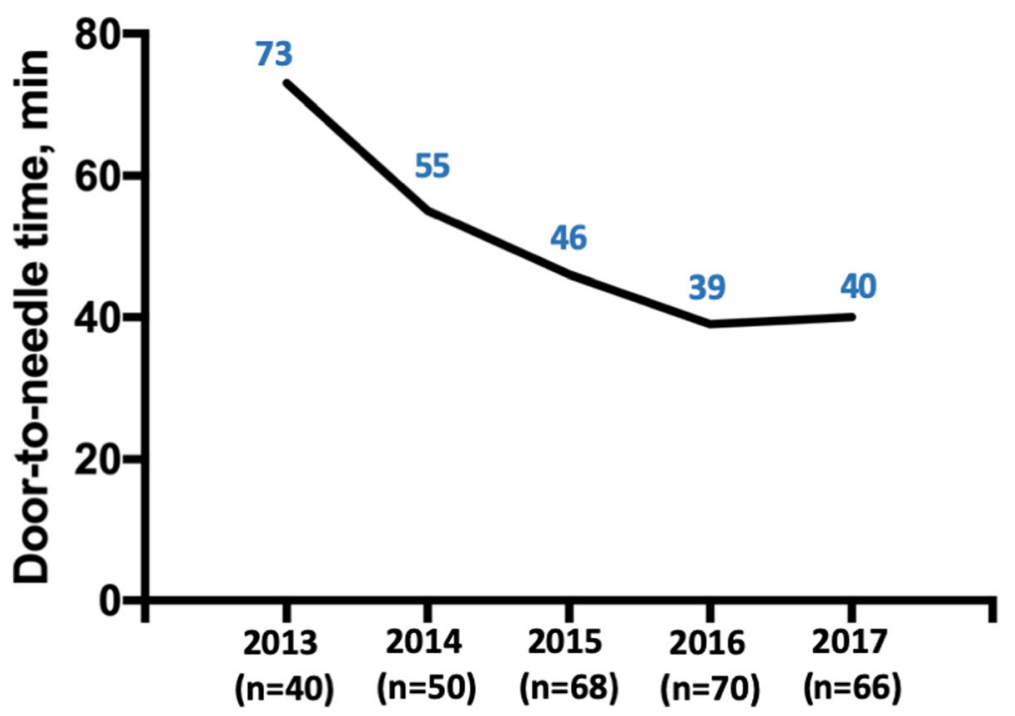

Fig. 1 Median door-to-needle time from 2013 to 2017

guidelines, IVT should be held until BP is less than 185/ $110 \mathrm{mmHg}$ [1]. In the pre-intervention group, severe hypertension was managed by the ER physicians. During post-intervention period, stroke team was managing hypertension in the $\mathrm{CT}$ suite and ED without any delay as soon as patient was deemed to be eligible for IVT. This simple change effectively minimized hypertensionrelated delay for IVT.

Waiting for blood testing results is another common reason for delay up to $60 \mathrm{~min}$ in some eligible patients $[5,18]$. Previous studies reported extremely low rates of unidentified coagulopathies and thrombocytopenia that would have been a contraindication for IVT [18-20]. Therefore, we implemented the initiative for IVT administration without waiting for blood test results unless patients were taking anticoagulants or had history of severe thrombocytopenia. There was no significant difference in the rate of $\mathrm{sICH}$ between pre-intervention and post-intervention groups. No patient suffered $\mathrm{sICH}$ from
IVT due to undiagnosed coagulopathy. In addition, the rates of $\mathrm{sICH}$ in our cohort were much lower than reported in clinical trials [2], confirming the safety of our simple initiative.

Another change we made in practice in January 2015 was to give IVT in the CT suite. As CT imaging is an indispensable diagnostic tool for decision-making for IVT, shortening the CT imaging-to-needle time may significantly improve DNT $[4,5,21,22]$. Our data confirmed that giving IVT in the CT suite minimizes the delay from CT to needle time without significant risk of complications.

Of note, the initial NIHSS scores in the postintervention group was significantly lower than that in the pre-intervention group. There were numerous possibilities to explain why more patients with non-disabling stroke (NIHSS $\leq 4)$ during the post-intervention group. Previous studies showed longer DNT in patients with minor stroke, possibly due to higher chance of atypical symptoms, delayed neurology notification and diagnosis $[23,24]$. The

Table 2 Outcomes in the pre- and post-intervention groups at discharge

\begin{tabular}{|c|c|c|c|c|c|c|}
\hline \multirow[t]{2}{*}{ Outcomes } & \multicolumn{2}{|c|}{ Patients receiving IVT } & \multirow{2}{*}{$\begin{array}{l}\text { OR } \\
(95 \% \mathrm{Cl})\end{array}$} & \multirow[t]{2}{*}{$p$ value } & \multirow{2}{*}{$\begin{array}{l}\text { Adjusted } \\
\text { OR } \\
(95 \% \mathrm{Cl})^{\mathrm{a}}\end{array}$} & \multirow[t]{2}{*}{$p$ value $^{a}$} \\
\hline & $\begin{array}{l}\text { Pre-intervention } \\
(n=90)\end{array}$ & $\begin{array}{l}\text { Post-intervention } \\
(n=136)\end{array}$ & & & & \\
\hline $\mathrm{sICH}$ & $1(1.1)$ & $2(1.5)$ & $\begin{array}{l}1.33 \\
(0.12-14.87)\end{array}$ & 0.817 & $\begin{array}{l}1.15 \\
(0.11-12.39)\end{array}$ & 0.911 \\
\hline mRS 0-1 & $21(23.3)$ & $40(29.4)$ & $\begin{array}{l}1.37 \\
(0.74-2.53)\end{array}$ & 0.314 & $\begin{array}{l}1.01 \\
(0.50-2.04)\end{array}$ & 0.983 \\
\hline mRS 5-6 & $39(43.3)$ & $34(25.0)$ & $\begin{array}{l}0.44 \\
(0.25-0.77)\end{array}$ & 0.004 & $\begin{array}{l}0.53 \\
(0.27-1.05)\end{array}$ & 0.068 \\
\hline mortality & $6(6.7)$ & $10(7.4)$ & $\begin{array}{l}1.11 \\
(0.39-3.17)\end{array}$ & 0.844 & $\begin{array}{l}1.33 \\
(0.44-4.03)\end{array}$ & 0.619 \\
\hline
\end{tabular}

Data are $\mathrm{n}(\%)$

sICH Symptomatic intracranial hemorrhage, OR Odds ratio, Cl Confidence interval, IVT Intravenous thrombolysis, mRS Modified Rankin Scale

${ }^{a}$ Adjusted for age, hypertension, diabetes, hyperlipidemia and baseline NIHSS 


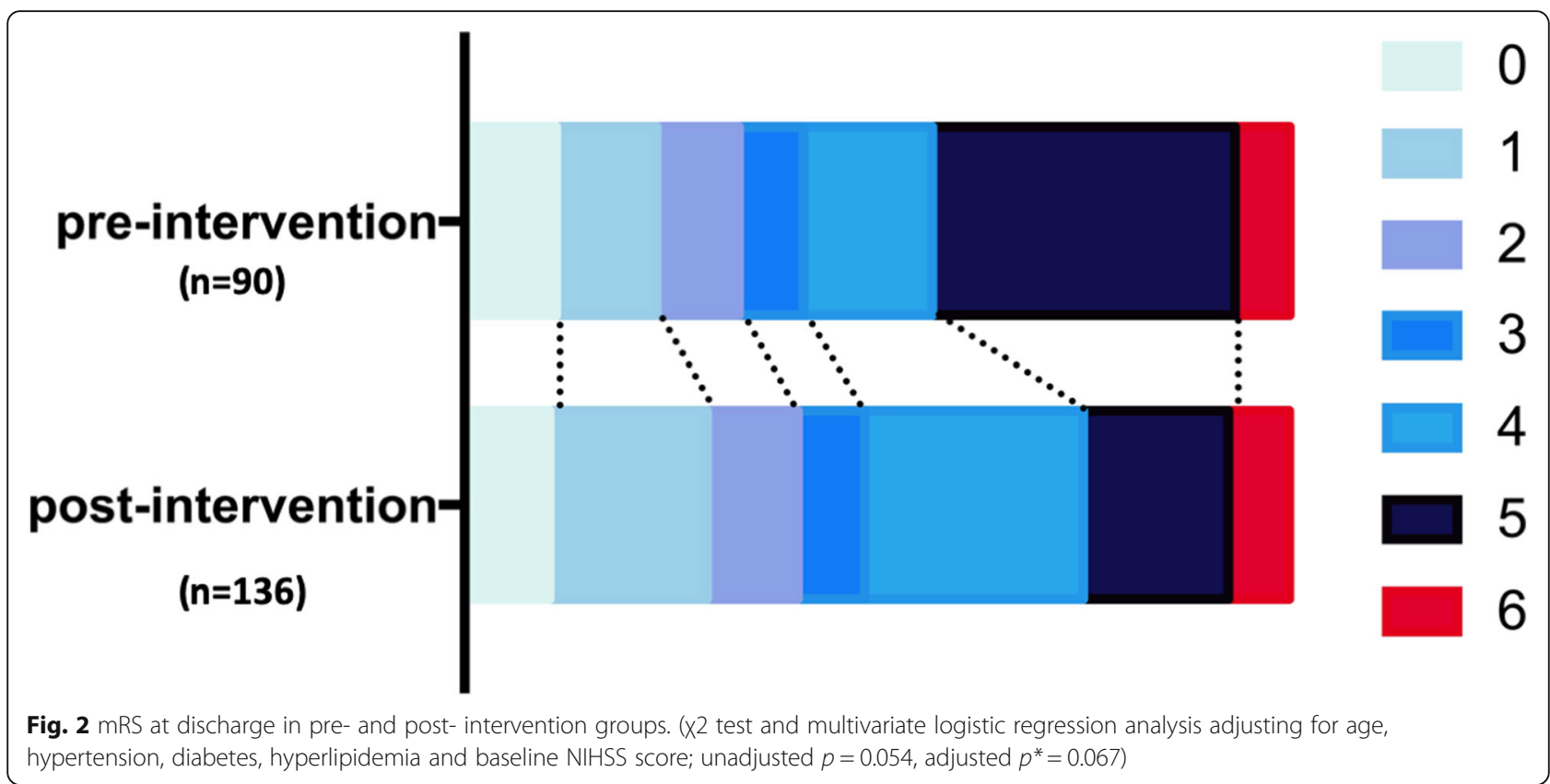

early diagnosis and decision making from better implementation of the Stroke: Target strategies in the postintervention group would naturally increase IVT for patients with minor strokes. In addition, better stroke aware, risk management and stroke prevention in recent years may have also decreased the ratio of patients with more severe stroke.

The strength of our study is the use of 3 easilyimplementable changes to reduce DNT without additional infrastructure cost or undue burden on the stroke team and ED staff. This simple initiative is effective, safe and easily replicable at other CSCs and PSCs in the U.S. and other countries.

This study has some limitations. First, it is a single center study. We used pre-intervention period as historic control. Our post-intervention data could be affected by unmeasurable confounding factors and gradual improvement in stroke care due to better training and experience. Second, although we had a one-year transition period for full implementation of the 3 changes, there might be incomplete adherence during the postintervention period. Since incomplete adherence to the changes would likely lead to prolonged DNT, it is possible that the simple changes could work better if the initiative was applied all the time effectively. Third, as the aim of this study is to investigate the effect of a simple quality improvement initiative on DNT for IVT, some factors associated with outcomes at discharged were not included (eg, early ischemic changes on imaging, large artery occlusions, thrombectomy treatment). Finally, we have not addressed other hurdles in delaying DNT, such as point of care coagulation testing for patients taking anticoagulants, moving directly from the EMS stretcher to CT scanner $[4,7,15]$. Additional easily-implementable changes at decentralized healthcare system may significantly reduce DNT at CSCs and PSCs in the U.S.

\section{Conclusions}

We demonstrated that three easily-implementable changes increase IVT rate and reduce DNT for IVT significantly without increasing the rate of IVT-related complications in our CSC.

\section{Abbreviations}

AHA: American Heart Association; AIS: Acute ischemic stroke; ASA: American Stroke emergency medical service; ER: Emergency room; IQR: Interquartile range; IVT: Intravenous thrombolysis; LKW: Last-known-well; mRS: Modified Rankin Scale; NIHSS: National Institutes of Health Stroke Scal; PSC: Primary stroke center; SD: Standard deviation; sICH: Symptomatic intracranial hemorrhage; tPA: Tissue-type plasminogen activator

\section{Acknowledgements}

Not applicable.

Authors' contributions

DT, ZZ: study design, data acquisition, analysis, interpretation, and drafting the manuscript. MS, HA: data interpretation and critical revision. DS: data acquisition. WY: study concept, design, data analysis, interpretation, and manuscript revision. All authors read and approved the final manuscript.

\section{Funding}

Not applicable.

Availability of data and materials

The data will be available from the corresponding author on reasonable request. 


\section{Ethics approval and consent to participate}

This study was approved by University of California Irvine Institutional Review Board, and informed consents were waived due to minimal risk of harm to the patients.

\section{Consent for publication}

Not applicable

\section{Competing interests}

The authors declare that they have no competing interests.

\section{Author details}

'Department of Neurology, University of California, Irvine, CA, USA. 2Department of Neurology, Huashan Hospital, Fudan University, Shanghai, China. ${ }^{3}$ Orange, USA.

Received: 14 August 2019 Accepted: 13 November 2019

Published online: 26 November 2019

\section{References}

1. Powers WJ, Rabinstein AA, Ackerson T, et al. 2018 guidelines for the early management of patients with acute ischemic stroke: a guideline for healthcare professionals from the american heart association/american stroke association. Stroke. 2018;49:e46-e110.

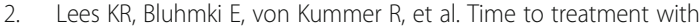
intravenous alteplase and outcome in stroke: an updated pooled analysis of ECASS, ATLANTIS, NINDS, and EPITHET trials. Lancet. 2010;375:1695-703.

3. Lindsberg PJ, Häppölä O, Kallela M, et al. Door to thrombolysis: ER reorganization and reduced delays to acute stroke treatment. Neurology. 2006;67:334-6.

4. Meretoja A, Strbian D, Mustanoja S, et al. Reducing in-hospital delay to 20 minutes in stroke thrombolysis. Neurology. 2012;79:306-13.

5. Meretoja A, Weir L, Ugalde M, et al. Helsinki model cut stroke thrombolysis delays to 25 minutes in Melbourne in only 4 months. Neurology. 2013;81: 1071-6.

6. Kamal N, Benavente O, Boyle K, et al. Good is not good enough: the benchmark stroke door-to-needle time should be 30 minutes. Can J Neurol Sci. 2014:41:694-6.

7. Kamal N, Holodinsky JK, Stephenson C, et al. Improving door-to-needle times for acute ischemic stroke: Effect of rapid patient registration, moving directly to computed. tomography, and giving alteplase at the computed tomography scanner. Circ Cardiovasc Qual Outcomes. 2017;10:e003242.

8. Van Schaik SM, Scott S, de Lau LM, et al. Short door-to-needle times in acute ischemic stroke and prospective identification of its delaying factors. Cerebrovasc Dis Extra. 2015:5:75-83.

9. Zinkstok SM, Beenen LF, Luitse JS, et al. Thrombolysis in stroke within 30 minutes: results of the acute brain care intervention study. PLoS One. 2016; $11: \mathrm{e} 0166668$.

10. Fonarow GC, Smith EE, Saver JL, et al. Improving door-to-needle times in acute ischemic stroke: the design and rationale for the american heart association/american stroke association's target: stroke initiative. Stroke. 2011:42:2983-9.

11. Certification Comprehensive Stroke Center. https://www.jointcommission. org/certification/advanced_certification_comprehensive_stroke_centers. aspx. Accessed 10 Aug 2019.

12. Kamal N, Sheng S, Xian Y, et al. Delays in door-to-needle times and their impact on treatment time and outcomes in get with the guidelines-stroke. Stroke. 2017;48:946-54

13. Xian $Y, X u H$, Lytle B, et al. Use of strategies to improve door-to-needle times with tissue-type plasminogen activator in acute ischemic stroke in clinical practice: findings from target: stroke. Circ Cardiovasc Qual Outcomes. 2017:10:e003227.

14. Man S, Zhao X, Uchino K, et al. Comparison of acute ischemic stroke care and outcomes between comprehensive stroke centers and primary stroke centers in the United States. Circ Cardiovasc Qual Outcomes. 2018;11: e004512.

15. Pihlasviita S, Mattila OS, Ritvonen J. Diagnosing cerebral ischemia with doorto-thrombolysis times below 20 minutes. Neurology. 2018;91:e498-508.

16. Raychev RI, Stradling D, Patel N, et al. Evolution of a U.S. County System for Acute Comprehensive Stroke Care. Stroke. 2018;49:1217-22.
17. Navalkele DD, Cai C, Vahidy F, et al. Higher prehospital blood pressure prolongs door to needle thrombolysis times: a target for quality improvement? Am J Emerg Med. 2016;34:1268-72.

18. Drescher MJ, Spence A, Rockwell D, et al. Point-of-care testing for coagulation studies in a stroke protocol: a time-saving innovation. Am J Emerg Med. 2011;29:82-5.

19. Rost NS, Masrur S, Pervez MA, et al. Unsuspected coagulopathy rarely prevents iv thrombolysis in acute ischemic stroke. Neurology. 2009;73:1957-62.

20. Cucchiara $B L$, Jackson $B$, Weiner $M$, et al. Usefulness of checking platelet count before thrombolysis in acute ischemic stroke. Stroke. 2007;38:1639-40.

21. Sauser $K$, Levine DA, Nickles AV, et al. Hospital variation in thrombolysis times among patients with acute ischemic stroke: the contributions of door-to-imaging time and imaging-to-needle time. JAMA Neurol. 2014;71: 1155-61.

22. Kelly AG, Hellkamp AS, Olson D, et al. Predictors of rapid brain imaging in acute stroke: analysis of the get with the guidelines-stroke program. Stroke. 2012:43:1279-84.

23. Tarnutzer AA, Lee SH, Robinson KA, et al. ED misdiagnosis of cerebrovascular events in the era of modern neuroimaging: a meta-analysis. Neurology. 2017;88:1468-77.

24. Yoo J, Sohn SI, Kim J, et al. Delayed intravenous thrombolysis in patients with minor stroke. Cerebrovasc Dis. 2018:46:52-8.

\section{Publisher's Note}

Springer Nature remains neutral with regard to jurisdictional claims in published maps and institutional affiliations.
Ready to submit your research? Choose BMC and benefit from:

- fast, convenient online submission

- thorough peer review by experienced researchers in your field

- rapid publication on acceptance

- support for research data, including large and complex data types

- gold Open Access which fosters wider collaboration and increased citations

- maximum visibility for your research: over $100 \mathrm{M}$ website views per year

At BMC, research is always in progress.

Learn more biomedcentral.com/submissions 